\title{
Evolutionary relationship between dinoflagellates bearing obligate diatom endosymbionts: insight into tertiary endosymbiosis
}

\author{
Yuji Inagaki, ${ }^{1,2}$ Joel B. Dacks, ${ }^{2}$ W. Ford Doolittle, ${ }^{2}$ Kazuo I. Watanabe ${ }^{3}$ \\ and Takeshi Ohama ${ }^{1}$
}

Author for correspondence: Yuji Inagaki. Tel: +1 902494 2968. Fax: + 19024941355. e-mail: yinagai@is.dal.ca

1 JT Biohistory Research Hall, 1-1 Murasaki-cho,

Takatsuki, Osaka

569-1125, Japan

2 Program in Evolutionary Biology, Canadian Institute for Advanced Research, Department of Biochemistry and Molecular Biology, Dalhousie University, Halifax, Nova Scotia, Canada B3H $4 \mathrm{H} 7$

3 Department of Biology, Faculty of Science, Osaka University, Toyonaka, Osaka 560-0043, Japan

\begin{abstract}
The marine dinoflagellates Peridinium balticum and Peridinium foliaceum are known for bearing diatom endosymbionts instead of peridinin-containing plastids. While evidence clearly indicates that their endosymbionts are closely related, the relationship between the host dinoflagellate cells is not settled. To examine the relationship of the two dinoflagellates, the DNA sequences of nuclear small-subunit rRNA genes (SSU rDNA) from Peridinium balticum, Peridinium foliaceum and one other peridinin-containing species, Peridinium bipes, were amplified, cloned and sequenced. While phylogenetic analyses under simple models of nucleotide substitution weakly support the monophyly of Peridinium balticum and Peridinium foliaceum, analyses under more sophisticated models significantly increased the statistical support for this relationship. Combining these results with the similarity between the two endosymbionts, it is concluded that (i) the two hosts have the closest sister relationship among dinoflagellates tested, (ii) the hypothesis that the diatom endosymbiosis occurred prior to the separation of the host cells is most likely to explain their evolutionary histories, and (iii) phylogenetic inferences under complex nucleotide evolution models seem to be able to compensate significant rate variation in the two SSU rDNA.
\end{abstract}

Keywords: Peridinium, plastid, SSU rDNA, among-site rate variation, nucleotide substitution model

\section{INTRODUCTION}

It is well established that plastids (or chloroplasts) in eukaryotes are derived either directly or indirectly from highly reduced endosymbiotic cyanobacteria. However, the evolution of extant photosynthetic eukaryotes is quite complex (reviewed by Gray, 1992; Gibbs, 1993; Delwiche \& Palmer, 1997; Bhattacharya \& Medlin, 1998; Cavalier-Smith, 1999; Delwiche, 1999). Plastids surrounded by double membranes, so

\footnotetext{
Abbreviations: Dist, distance; indels, insertions/deletions; ML, maximum likelihood; ML-Dist, maximum-likelihood distance; MP, maximum-parsimony; SSU rDNA, small-subunit rRNA gene; $-\ln \mathrm{L}$, log likelihood; $P_{\mathrm{INV}}$ proportion of invariable sites.

The GenBank accession numbers for the Peridinium balticum, Peridinium foliaceum and Peridinium bipes SSU rDNA sequences are AF231803, AF231804 and AF231805, respectively.
}

called 'primary' plastids, are found in green algae, land plants, red algae and glaucocystophytes. These primary plastids are believed to be evolved via direct eukaryote-cyanobacterium endosymbiosis. It remains unclear whether plastids in the four lineages are direct descendants of a single endosymbiotic event. However, recent evidence supports the monophyly of green algae (plus land plants) and red algae, with weaker evidence pointing also to a sister relationship with glaucocystophytes (Moreira et al., 2000).

'Secondary' plastids, be they triple or quadruple membrane bound, are found in as widely divergent eukaryotic lineages as euglenoids, apicomplexan parasites, chromist algae (heterokonts, haptophytes, cryptomonads and chlorarachniophytes) and dinoflagellates. This type of plastid is presumed to be derived from a photosynthetic eukaryote bearing primary 
plastids that was engulfed by a heterotrophic eukaryote through phagocytosis (or myzocytosis). Chlorarachniophytes and cryptomonads provide direct evidence for this hypothesis (reviewed by Gilson \& McFadden, 1997); remnants of endosymbiotic nuclei (nucleomorphs) and plastids are found in the periplastidal cytoplasm that is separated from the host cytosol by two membranes. These cell structures have been interpreted as intermediates in the reduction of the engulfed photosynthetic eukaryotes into secondary plastids without nucleomorphs. Recent molecular phylogenetic studies have demonstrated that eukaryotes bearing secondary plastids usually have heterotrophic relatives, and they are most probably the result of the independent acquisition of their plastids after the separation of the photosynthetic lineage from their heterotrophic sisters (Van de Peer et al., 1996; Inagaki et al., 1997, 1998; Van der Auwera et al., 1998).

In addition, several dinoflagellates maintain chromist algae, which themselves have secondary plastids, as endosymbionts (Delwiche, 1999). Such 'tertiary' endosymbioses are not as well studied, but the marine dinoflagellates Peridinium balticum and Peridinium foliaceum are well-established cases. The endosymbionts of Peridinium balticum and Peridinium foliaceum are remarkably similar in their structural features and pigment composition (reviewed by Chesnick et al., 1997). The endosymbionts are separated from the host cytosol by a single membrane, presumably having lost their cell wall (Dodge, 1971; Tomas et al., 1973), and the endosymbiont of Peridinium balticum undergoes amitotic cell division (Tippit \& Pickett-Heaps, 1976). These observations strongly suggest that the algae are not the transient association of the host cells with an autonomous photosynthetic eukaryote. Given this, it is surprising that the endosymbionts appeared to retain their mitochondria as well as eukaryotic nucleus and plastids (Dodge, 1971; Tomas et al., 1973).

Based on the photosynthetic pigmentation and recent phylogenetic study of the nuclear small-subunit rRNA (SSU rDNA) of the two endosymbionts, it was clearly shown that the two endosymbionts are closely related, and descendants of pennate diatoms (family Bacillariaceae) (Dodge, 1971; Tomas et al., 1973; Chesnick et al., 1997). The phylogenetic analysis inferred from a plastid gene, $r b c L$ (encoding the large subunit of ribulose-1,5-bisphosphate carboxylase/oxygenase), also indicated that the endosymbiont of Peridinium foliaceum has a diatom origin (Chesnick et al., 1996). Curiously, despite the ultrastructural similarities of the two hosts, Peridinium balticum and Peridinium foliaceum, their isozyme profiles are significantly different (Whitten \& Hayhome, 1986). These conflicting data about their relationship make it difficult to determine whether the obligate diatom endosymbiosis occurred before or after the diversification of the host lineages.

To examine the phylogenetic positions of Peridinium balticum and Peridinium foliaceum among dinoflagel- lates, we amplified, cloned and sequenced their (nuclear) SSU rDNA sequences, as well as the sequence of an additional peridinoid species bearing a standard (peridinin-containing) dinoflagellate plastid, Peridinium bipes. Our exhaustive phylogenetic analyses inferred from SSU rDNA sequences succeeded in recovering a closest sister relationship between Peridinium balticum and Peridinium foliaceum, and we conclude that the ancestor of the two dinoflagellates engulfed a pennate diatom.

\section{METHODS}

Amplification, cloning and sequencing of SSU rDNA. Peridinium balticum (CS-38) and Peridinium foliaceum (LB1688) were purchased from the CSIRO division of Marine Research, Australia (Castray Esplanade, Hobart, Tasmania 7000, Australia). Peridinium bipes (NIES 364) was purchased from the National Institute for Environmental Studies, Japan (16-2 Onogawa, Tsukuba, Ibaragi 305-0053, Japan). Cells were cultured with the medium recommended by the suppliers with $50 \mathrm{mg}$ gentamicin $\mathrm{ml}^{-1}$ to prevent bacterial growth. One gram of frozen cells was ground into a fine powder using a bead-mill and resuspended in one volume of CTAB extraction buffer $[2 \%(\mathrm{w} / \mathrm{v})$ cetyltrimethylammonium bromide (CTAB), $100 \mathrm{mM}$ Tris $/ \mathrm{HCl}(\mathrm{pH} 8 \cdot 0), 20 \mathrm{mM}$ EDTA (pH 8.0), 1.4 M NaCl]. Nucleic acids were extracted twice with chloroform following incubation for $1 \mathrm{~h}$ at $65^{\circ} \mathrm{C}$, and then precipitated by addition of an equal volume of CTAB precipitation buffer $[1 \%$ (w/v) CTAB, $50 \mathrm{mM}$ Tris/ $\mathrm{HCl}(\mathrm{pH} \mathrm{8} 0), 10 \mathrm{mM}$ EDTA (pH 8.0)]. The resultant pellet was resuspended in high-salt buffer $[10 \mathrm{mM}$ Tris $/ \mathrm{HCl}$ $(\mathrm{pH} 8 \cdot 0), 0 \cdot 1 \mathrm{mM}$ EDTA $(\mathrm{pH} 8 \cdot 0), 1 \mathrm{M} \mathrm{NaCl}$. After 2propanol precipitation, nucleic acids were dissolved in distilled water and used for PCR.

A pair of synthesized primers, 5'-TACCTGGTTGATCC TGCCAGTA-3' and 5'-CCATCCGCAGGTTCACCTCA$3^{\prime}$, was used to amplify nearly the entire SSU rDNA. PCR was carried out as follows: denaturation at $94{ }^{\circ} \mathrm{C}$ for $15 \mathrm{~s}$, annealing at $60{ }^{\circ} \mathrm{C}$ for $1 \mathrm{~min}$ and extension at $72{ }^{\circ} \mathrm{C}$ for $2 \mathrm{~min}$ for 30 cycles. The amplified fragments were subsequently ligated into the pT7Blue T-vector (Novagen) and transformed into Escherichia coli JM109. DNA sequences were determined for both strands by cycle sequencing with dye-terminators using a DNA sequencer model 377 (Applied Biosystems).

Phylogenetic analyses. The three Peridinium rDNA sequences were manually aligned along with the previously published sequences of 49 dinoflagellates and two apicomplexan parasites (Perkinsus sp., L07375; Toxoplasma gondii, X65508) using MACCLADE version 4.0b12 (Maddison \& Maddison, 2000). For alignment editing, we referred to the secondary structure of SSU rRNA molecule deposited in the rRNA WWW Server at the University of Antwerp (http:// rrna.uia.ac.be/index.html). After polishing of the alignment by eye and exclusion of all ambiguous sites, it included 54 taxa with 1723 sites. Preliminary phylogenetic analyses were performed on this dataset under maximum-parsimony (MP) optimality criteria. Subsequently we generated an additional two datasets for the later analyses.

A second alignment comprising 40 taxa and 1723 sites was generated by removing redundant taxa (i.e. taxa in the clades with bootstrap scores of 100 in MP analyses). This dataset was tested for optimal fit of various models of nucleotide 
evolution using MODELTEST version 3.0b3 (Posada \& Crandall, 1998). The proportion of invariable sites $\left(P_{\text {INV }}\right)$, a discrete $\gamma$ distribution (four categories) and base frequencies were similarly estimated from the dataset. A maximumlikelihood distance (ML-Dist) bootstrap analysis (100 resampling) under an optimal model of nucleotide substitution incorporating the $P_{\mathrm{INV}}$ and a discrete $\gamma$ distribution with the empirical base frequencies was performed. For each replicate, starting trees were constructed by 100 random additions.

The third dataset of 22 taxa and 1718 sites was subjected to analysis using MODELTEST (Posada \& Crandall, 1998), and analysed using maximum-likelihood (ML) and distance (Dist) methods under two optimal models recommended as well as three simple models. Bootstrap analyses (100 resampling) using the Dist method under the five models were performed. ML bootstrap analyses under the two optimal models (100 resamplings) were operated without branchswapping (the 'Fast' stepwise-addition option in PAUP* version 4.0b3; Swofford, 1998) to save computational time. For all phylogenetic analyses in this study, PAUP* version 4.0b3 was used (Swofford, 1998).

\section{RESULTS}

\section{The Peridinium SSU rDNA sequences}

The amplified regions of SSU rDNA for Peridinium bipes, Peridinium balticum and Peridinium foliaceum were 1796, 1796 and $1795 \mathrm{bp}$, respectively. G+C contents are 48,47 and $46 \mathrm{~mol} \%$, respectively. The partial sequence of the previously reported SSU rDNA for Peridinium foliaceum (M88517) was identical to the sequence determined in this study. The number of nucleotide substitutions and single nucleotide insertions/deletions (indels) between the Peridinium balticum and Peridinium foliaceum genes were 145 and 4 , respectively (Table 1), while the endosymbiont SSU rDNAs have only $1 \%$ difference (18 substitutions out of 1788 sites; Chesnick et al., 1997). No difference between the putative secondary structures of the Peridinium balticum and Peridinium foliaceum rDNAs was detected (data not shown). Based on their structures, 103 out of 145 substitutions and two of the indels corresponded to non-conserved regions. Comparing

Table 1. Nucleotide substitution matrix of the four Peridinium SSU rDNA and number of unique substitutions

Pfol, Peridium foliaceum; Pbal, Peridinium balticum; Pbip, Peridinium bipes; Psp., Peridinium sp. Number of single nucleotide insertions/deletions are indicated in parentheses.

\begin{tabular}{|lcccc|}
\hline & Pbal & Pbip & Psp. & Unique substitutions* \\
\hline Pfol & $145(4)$ & $223(0)$ & $233(1)$ & 73 \\
Pbal & & $175(2)$ & $141(5)$ & 34 \\
Pbip & & & $108(1)$ & 28 \\
Psp. & & & & 36 \\
\hline
\end{tabular}

* Includes unique insertions/deletions. the four Peridinium SSU rDNA, we measured the number of unique substitutions among 1730 sites in each sequence (Table 1). Interestingly, the Peridinium foliaceum gene appeared to have 73 unique substitutions, almost twice that observed in the other three taxa (Table 1). These observations strongly imply that the Peridinium foliaceum SSU rDNA evolves almost twice as fast as the Peridinium balticum gene.

\section{Phylogenetic analyses}

In preliminary MP analyses, Peridinium balticum and Peridinium foliaceum were positioned as sister taxa (bootstrap score, 35; data not shown). Corresponding to the outstanding number of the unique substitutions found in the Peridinium foliaceum gene (Table 1), the Peridinium foliaceum branch was twice as long as that of Peridinium balticum in the most parsimonious trees (data not shown, but the same results were observed in Fig. 1). Recently it has been shown that phylogenetic inference without accounting for among-site rate variation can lead to artefactual results (e.g. Silberman et al., 1999; Stiller \& Hall, 1999). Because the difference in substitution rate between the SSU rDNA sequences of interest may mask phylogenetic signal, it is possible that our MP analyses may not be able to robustly reconstruct relationships.

A subdataset (40 taxa/1723 sites), which does not include redundant taxa, was analysed using MODELTEST (Posada \& Crandall, 1998). Through calculation of $\log$-likelihood $(-\ln \mathrm{L})$ scores, this program found that a TrN model of nucleotide evolution (Tamura \& Nei, 1993) incorporating the proportion of invariable sites and a discrete $\gamma$ distribution (four categories) ( $\mathrm{TrN}+$ $\left.P_{\mathrm{INV}}+\gamma\right)$ was significantly better than other models examined. The dataset was therefore analysed using a ML-Dist method under this model (Fig. 1a). For the most part, the resultant tree corresponds to the general classification and previous investigations of dinoflagellate phylogeny based on SSU rDNA sequences (e.g. Grzebyk et al., 1998; Montresor et al., 1999). Our analysis showed weak affinity for the Peridinium balticum-Peridinium foliaceum clade and strong support for the Peridinium bipes-Peridinum sp. relationship (bootstrap scores, 54 and 100, respectively; Fig. 1a).

Considering computational time, for this set of analyses, a smaller dataset (20 taxa/1718 sites) was generated. MODELTEST (Posada \& Crandall, 1998) found that TrN (Tamura \& Nei, 1993) and general-time-reversible (GTR) models (Rodríguez et al., 1990) incorporating the $P_{\text {INV }}$ and a discrete $\gamma$ distribution best describe the dataset $(-\ln \mathrm{L}$ scores estimated under the two models are almost the same, 10782). The $-\ln \mathrm{L}$ scores under the simple models, JC (Jukes \& Cantor, 1969), F81 (Felsenstein, 1981) and K2P (Kimura, 1980), that incorporated neither $P_{\mathrm{INV}}$ and a discrete $\gamma$ distribution, were poor $(-\ln \mathrm{L}=12146,12126$ and 11811 , respectively). Phylogenies inferred from the third dataset matched the overall topology of those in the first two 

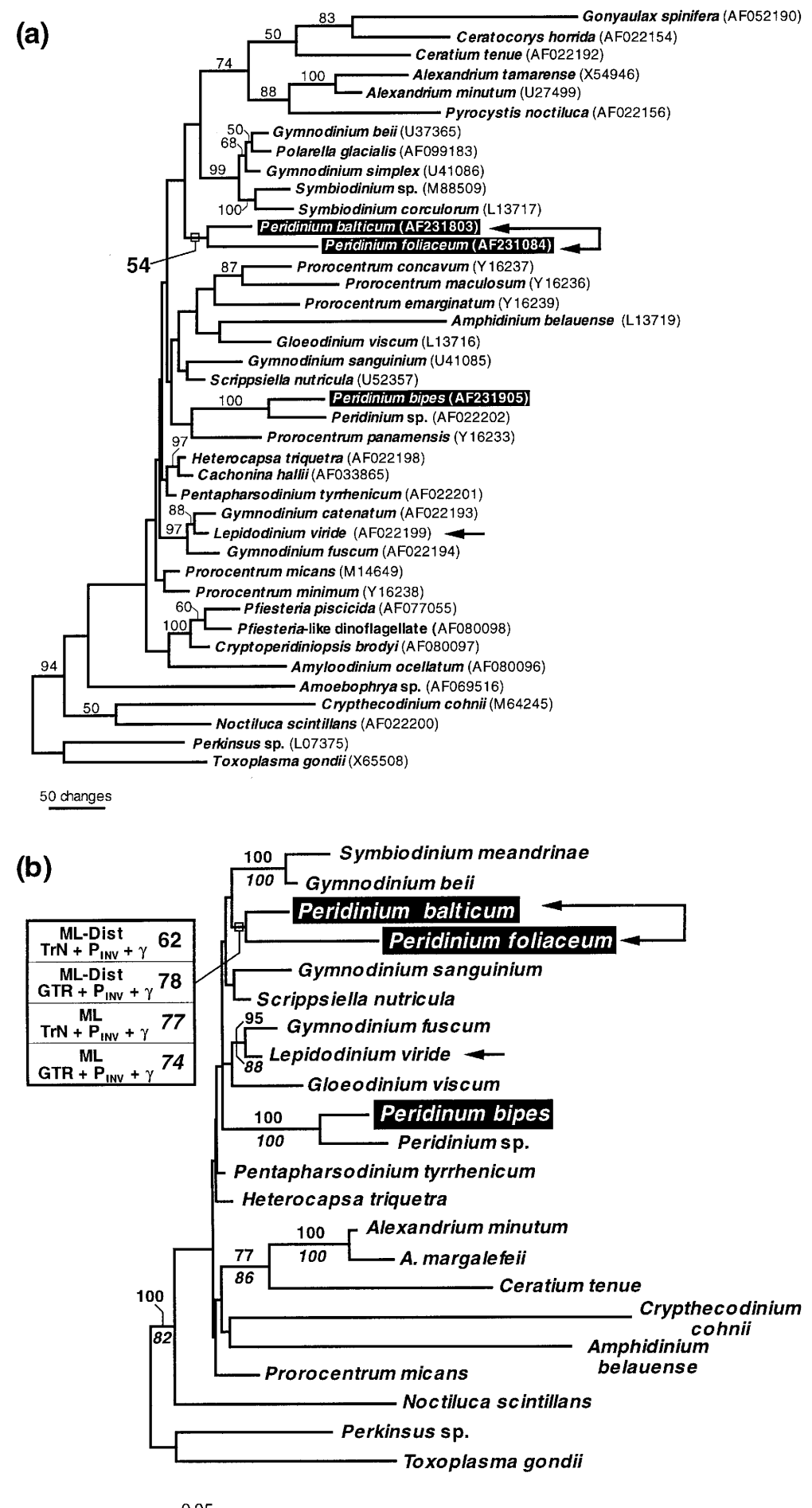

\begin{abstract}
Fig. 1. Phylogenetic trees inferred from dinoflagellate SSU rDNA sequences. (a) The maximum-likelihood distance (ML-Dist) tree (40 taxa/1723 sites) reconstructed under a TrN nucleotide substitution model (Tamura $\&$ Nei, 1993) incorporating the proportion of invariable sites $\left(P_{\mathrm{INV}}\right)$ and a discrete $\gamma$ distribution (four categories) $\left(\operatorname{TrN}+P_{\mathrm{INV}}+\gamma\right)$. For the bootstrap analyses, 100 replicates of the original dataset were used, with starting trees for each replicate constructed by 100 random addition. Only bootstrap scores above $50 \%$ are indicated. (b) The best maximum-likelihood (ML) tree inferred from the dataset (20 taxa/1718 sites) under a $\operatorname{TrN}+P_{\text {INV }}+\gamma$ model. The bootstrap scores indicated in roman and italic were calculated by ML-Dist and ML methods under a $\operatorname{TrN}+P_{\mathrm{INV}}+\gamma$ model (100 resamplings), respectively. Only bootstrap score above $50 \%$ are indicated. For the Peridinium balticum-Peridinium foliaceum clade, bootstrap scores obtained from ML-Dist and ML analyses under a general-time-reversible (GTR) model (Rodríguez et al., 1990) incorporating the $P_{\text {INV }}$ and a discrete $\gamma$ distribution (four categories) are also indicated. ML bootstrap analyses were conducted with no branch-swapping using the 'Fast' stepwise-addition option in PAUP* version 4.0b3 (Swofford, 1998). Lineages bearing tertiary plastid are highlighted by arrows. The Gymnodinium breve, Gymnodinium galatheanum and Gymnodinium aureolum SSU rDNA are not included in these analyses.
\end{abstract}

(Fig. 1b). For the Peridinium balticum-P. foliaceum clade, bootstrap scores of 62 and 78 were obtained using ML-Dist methods under $\operatorname{TrN}+P_{\mathrm{INV}}+\gamma$ and GTR $+P_{\mathrm{INV}}+\gamma$ models, respectively. The ML bootstrap analyses under those two optimal models gave bootstrap scores of 77 and 74 for the same clade (Fig. $1 \mathrm{~b}$; Table 2). All of the top $100 \mathrm{ML}$ trees reconstructed under the optimal models included the Peridinium
balticum-Peridinium foliaceum clade (data not shown). However, neither Dist or ML analyses under the simple models, JC, F81 and K2P, supported this clade (Table 2). While the Peridinium bipes-Peridinium sp. clade was solid irrespective of the methods or models (Table 2), we failed to detect any connection between the Peridinium balticum-Peridinium foliaceum and Peridinium bipes-Peridinium sp. clades (Fig. 1). 
Table 2. Impact of nucleotide substitution and among-site rate variation models on dinoflagellate phylogeny

Symbols/abbreviations: + , the best ML tree includes the topology of interest; - , the bootstrap consensus tree or best ML tree does not have the topology of interest; ND, bootstrap analysis using ML method under JC, F81 or K2P model was not done. ML bootstrap analyses were conducted with no branch-swapping (PAUP* version 4.0b3; Swofford, 1998).

\begin{tabular}{|c|c|c|c|c|c|c|}
\hline & \multirow[t]{2}{*}{ Criteria } & \multicolumn{5}{|c|}{ Model } \\
\hline & & $\mathrm{JC}$ & F81 & K2P & $\operatorname{TrN}+P_{\mathrm{INV}}+\gamma$ & GTR $+P_{\mathrm{INV}}+\gamma$ \\
\hline \multirow[t]{3}{*}{ Peridinium balticum-Peridinium foliaceum clade } & Dist (bootstrap) & - & - & - & 62 & 78 \\
\hline & ML (bootstrap) & ND & ND & ND & 77 & 74 \\
\hline & best ML tree & - & - & - & + & + \\
\hline \multirow[t]{3}{*}{ Peridinium bipes-Peridinium $\mathrm{sp}$. clade } & Dist (bootstrap) & 100 & 100 & 100 & 100 & 100 \\
\hline & ML (bootstrap) & ND & ND & ND & 100 & 100 \\
\hline & best ML tree & + & + & + & + & + \\
\hline \multirow[t]{3}{*}{ Basal branching of Gonyaucales and Nocticales } & Dist (bootstrap) & 79 & 74 & 74 & - & - \\
\hline & ML (bootstrap) & ND & ND & $\mathrm{ND}$ & - & - \\
\hline & best ML tree & + & + & + & - & - \\
\hline
\end{tabular}

\section{DISCUSSION}

\section{Impact of nucleotide evolution models on dinoflagellate SSU rDNA phylogeny}

Our SSU rDNA analyses reconstruct major dinoflagellate clades, i.e. the clades comprised of the species belonging to the families Gonyaulacales, the Symbiodinium and Gymnodinium species, the two Gymnodinium species and Lepidodinium viride (Fig. 1), in agreement with other previous studies (e.g. Grzebyk et al., 1998; Montresor et al., 1999). In phylogenetic inferences under simple nucleotide substitution models without accounting for among-site rate variation, species belonging to the families Gonyaulacales and Nocticales, such as Alexandrium minutum, Alexandrium margalefeii, Ceratium tenue, Crypthecodinium cohnii, Amphidinium belauense and Noctiluca scintillans, were found as basal branches among dinoflagellates (Table 2). However, in our phylogenies reconstructed under the complex models, which best described our SSU rDNA dataset, there is no statistical support for relative branching order between the robust clades that we obtained, nor for the deeply diverging branches in our trees (Fig. 1; Table 2). Considering their long branch lengths, we suspect the positions of some of the basal diverging dinoflagellate sequences, in particular the family Gonyaulacales, may be artefactual, resulting from 'long branch attraction'. Our analyses suggest that the complex models seem less susceptible to artificial resolution due to long branch attraction as shown by Silberman et al. (1999).

\section{'Single-endosymbiosis' versus 'separate- endosymbiosis'}

Despite both morphological and molecular evidence strongly indicating a close relationship between the diatom endosymbionts of Peridinium balticum and Peridinium foliaceum, the available data on the dino- flagellate hosts themselves are not sufficient to resolve their relationships (reviewed by Chesnick et al., 1997). This relationship is the key to the history of plastid acquisition in these two Peridinium species. So far, isozyme profile (Whitten \& Hayhome, 1986) is consistent with the 'separate-endosymbiosis' hypothesis that the two dinoflagellates captured the same or a similar diatom species independently. However, no molecular sequence data have been available for both Peridinium balticum and Peridinium foliaceum host cells, and statistical examinations of their relationship were therefore impossible. If a phylogenetic affinity between the two hosts was statistically supported, the 'single-endosymbiosis' hypothesis - that the ancestor of two dinoflagellates engulfed a diatom would be favoured over the 'separate-endosymbiosis' hypothesis.

Preliminary MP analyses lent no support to either acquisition scenarios of the diatom endosymbionts in Peridinium balticum and Peridinium foliaceum. However, as these analyses did not account for rate heterogeneity among sites, more stringent analyses with biologically relevant models for nucleotide evolution were performed. Analyses using ML-Dist and ML methods under the complex models of nucleotide evolution significantly improved the statistical support for the Peridinium balticum-Peridinium foliaceum clade (Table 2). These results are in contrast to inferences under simple models without consideration of amongsite rate variation (Table 2). Difficulty in resolving the relationship between Peridinium balticum and Peridinium foliaceum is most probably caused by the variation of nucleotide substitution rates between the two SSU rDNA, and thus biologically relevant nucleotide evolution models should be adopted to deal with this problematic dataset. Since these models were found to best describe the dataset, we conclude that Peridinium balticum and Peridinium foliaceum are the closest relatives among the species examined. This is in 
agreement with morphological comparisons of relevant cell structure (reviewed by Chesnick et al., 1997).

Large indels in SSU rDNA are presumed to accelerate the substitution rate over the entire gene in order to preserve overall rRNA structure (Stiller \& Hall, 1999). Unfortunately, comparison between the putative secondary structures of the Peridinium balticum and Peridinium foliaceum SSU rDNA suggests no such indels (data not shown). We can see no obvious molecular or biological rationale for the rate acceleration of the Peridinium foliaceum SSU rDNA. Further sequencing and comparison of the Peridinium balticum and Peridinium foliaceum protein-coding genes are necessary to examine whether the rate acceleration extends to the entire nuclear genome of Peridinium foliaceum. If so, this might reconcile their isozyme profiles (Whitten \& Hayhome, 1986) that seem contradictory to the conclusions reached by morphological studies and our molecular analyses. Assembled, these data lead us to prefer the scenario that a common ancestor of the two dinoflagellates engulfed a pennate diatom.

Nevertheless, the relationship between Peridinium balticum and Peridinium foliaceum still needs to be tested by SSU rDNA phylogeny with a broader taxon sampling. If some species with peridinin-containing plastids directly clusters with either Peridinium balticum or Peridinium foliaceum in a future study, the 'single-endosymbiosis' scenario will be disfavoured.

\section{Frequency of tertiary endosymbiosis in dinoflagellate evolution}

When and how many times tertiary endosymbioses have occurred during dinoflagellate evolution is an intriguing question. The relative positions of the tertiary plastid-containing dinoflagellates might give some indication of the number of times these events have occurred. Unfortunately, the relative position of the Peridinium balticum-Peridinium foliaceum clade in the dinoflagellate phylogeny is totally unresolved; this clade showed no affinity to other species including Peridinium sp. and Peridinium bipes (Fig. 1). KishinoHasegawa tests rejected neither the monophyly of the four Peridinium species nor the basal separation of the Peridinium balticum-Peridinium foliaceum clade (data not shown). However, the triple-enveloped eyespots in the cytosols of Peridinium balticum and Peridinium foliaceum have been described, and presumed to be vestiges of plastids surrounded by three membranes (Horiguchi \& Pienaar, 1991). Therefore, the ancestor of the two dinoflagellates may have replaced the original plastid with a diatom endosymbiont, arguing against the diatom endosymbiosis as the basal condition for the dinoflagellate clade.

Recently, the three marine dinoflagellates Dinothrix paradoxa, Gymnodinium quadrilobatum and Peridinium quinquecorne have been described as bearing diatom endosymbionts (Horiguchi \& Pienaar, 1991, 1994; Horiguchi \& Chihara, 1993). Unfortunately, whether these diatoms are permanent residents is unclear. Cavalier-Smith \& Lee (1985) make the distinction between transient endosymbiont and permanent resident (organelle) based on extent of hostendosymbiont integration. Organelles transfer some of their genes to their host's nucleus, and possess the molecular machinery to translocate the proteins encoded by such genes. Searching the nuclear genomes of diatom-bearing dinoflagellates for genes that are unambiguously derived from the diatom endosymbiont might clarify the question of whether the endosymbionts deserve true organelle status. The relationships among the dinoflagellates bearing diatom endosymbionts, including Peridinium balticum and Peridinium foliaceum, and their relative positions in dinoflagellate phylogeny are important in estimating the frequency of tertiary endosymbiosis between diatoms and dinoflagellates.

The extant plastid in the dinoflagellate Lapidodinium viride is most probably acquired by plastid replacement via tertiary endosymbiosis (reviewed by Delwiche, 1999). This dinoflagellate possesses a green-pigmented plastid surrounded by four membranes. In our analyses, Lapidodinium robustly clustered with Gymnodinium catenatum and Gymnodinium fuscum, which have peridinin-containing plastids (Fig. 1). Therefore, plastid replacement occurred after the separation of Lapidodinium and the two Gymnodinium species. Other tertiary plastids, assumed to be originated from haptophytes, have been reported in Gymnodinium breve, Gymnodinium galatheanum and Gymnodinium aureolum (Delwiche, 1999). Then we presume that tertiary endosymbioses (and plastid-replacement) have occurred at least three times during dinoflagellate evolution (highlighted by arrows in Fig. 1; Gymnodinium breve, Gymnodinium galatheanum and Gymnodinium aureolum are not included).

Dinoflagellates are easily maintained in the laboratory, are experimentally tractable, and have had a complex and varied history of plastid acquisition. They therefore make excellent models for the investigation of tertiary endosymbiosis. In order to best study this fascinating problem, however, a robust phylogeny of dinoflagellates based both on biologically relevant models of evolution for SSU rDNA as well as analyses from protein-coding genes are crucial.

\section{ACKNOWLEDGEMENTS}

We thank M. Ehara (Kobe University, Japan) for valuable discussions and comments on the previous version of this manuscript. We also thank J. M. Archibald, A. Lohan and A. J. Roger (Dalhousie University, Canada) for critical comments on this manuscript. Y.I. and K.I.W. are supported by a Research Fellowship of the Japanese Society for Promotion of Science for Young Scientists in Abroad. J.B.D. is supported by a Walter C. Sumner Memorial Fellowship and a studentship awarded to W.F.D. by the Canadian Medical Research Council. W.F.D. is supported by grant ML4465 from the Canadian Medical Research Council. A visiting associate professor position was arranged 
for T.O. at Osaka University in order to permit collaboration with postgraduates and use of the various facilities.

\section{REFERENCES}

Bhattacharya, D. \& Medlin, L. (1998). Algal phylogeny and the origin of land plants. Plant Physiol 116, 9-15.

Cavalier-Smith, T. (1999). Principles of protein and lipid targeting in secondary symbiogenesis: euglenoid, dinoflagellates, and sporozoan plastid origins and the eukaryotic family tree. $J$ Eukaryot Microbiol 46, 347-366.

Cavalier-Smith, T. \& Lee, J. L. (1985). Protozoa as hosts for endosymbioses and the conversion of symbionts into organelles, J Protozool 32, 376-379.

Chesnick, J. M., Morden, C. W. \& Schmieg, A. M. (1996). Identity of the Peridinium foliaceum (Pyrrhophyta) endosymbiont: analysis of the rbcLS operon. $J$ Phycol 32, 850-857.

Chesnick, J. M., Hooistra, W. H., Wellbrock, U. \& Medlin, L. K. (1997). Ribosomal RNA analysis indicates a benthic pennate diatom ancestry for the endosymbionts of the dinoflagellates Peridinium foliaceum and Peridinium balticum (Pyrrhophyta). $J$ Eukaryot Microbiol 44, 314-320.

Delwiche, C. F. (1999). Tracing the thread of plastid diversity through the tapestry of life. Am Nat 154 (suppl.), S164-S177.

Delwiche, C. F. \& Palmer, J. D. (1997). The origin of plastids and their spread via secondary symbiosis. Plant Syst Evol $\mathbf{5 3}$ (suppl.), 53-86.

Dodge, J. D. (1971). A dinoflagellate with both a mesokaryotic and a eucaryotic nucleus. Protoplasma 73, 145-157.

Felsenstein, J. (1981). Evolutionary trees from DNA sequences: a maximum likelihood approach. J Mol Evol 17, 368-376.

Gibbs, S. P. (1993). The evolution of algal chloroplasts. In Origins of Plastids, pp. 107-121. Edited by R. A. Lewin. New York: Chapman \& Hall.

Gilson, P. R. \& McFadden, G. I. (1997). Good things in small packages: the tiny genomes of chlorarachniophyte endosymbionts. Bioessays 19, 167-173.

Gray, M. W. (1992). The endosymbiont hypothesis revisited. Int Rev Cytol 141, 233-357.

Grzebyk, D., Sako, Y. \& Berland, B. (1998). Phylogenetic analysis of nine species of Prorocentrum (Dinophyceae) inferred from $18 \mathrm{~S}$ ribosomal DNA sequences, morphological comparisons and description of Prorocentrum panamensis, sp. nov. J Phycol 34, 1055-1068.

Horiguchi, T. \& Chihara, M. (1993). Life cycle and ultrastructure of Dinothrix paradoxa (Dinophyceae). The 15th International Botanical Congress, Yokohama, Japan, August-September 1993, p. 216 (abstract).

Horiguchi, T. \& Pienaar, R. N. (1991). Ultrastructure of a marine dinoflagellate, Peridinium quinquecorne Abé (Peridiniales) from South Africa with particular reference to its chrysophyte endosymbiont. Bot Mar 34, 123-131.

Horiguchi, T. \& Pienaar, R. N. (1994). Ultrastructure of a new marine sand dwelling dinoflagellate, Gymnodinium quadrilobatum sp. nov. (Dinophyceae) with special reference to its endosymbiotic alga. Eur J Phycol 29, 237-245.

Inagaki, Y., Hayashi-Ishimaru, Y., Ehara, M., Igarashi, I. \& Ohama, T. (1997). Algae or protozoa: phylogenetic position of eugleno- phytes and dinoflagellates as inferred from mitochondrial sequences. J Mol Evol 45, 295-300.

Inagaki, Y., Ehara, M., Watanabe, K. I., Hayashi-Ishimaru, Y. \& Ohama, T. (1998). Directionally evolving genetic code: the UGA codon from stop to tryptophan in mitochondria. J Mol Evol 47, 378-384.

Jukes, T. H. \& Cantor, C. R. (1969). Evolution of protein molecules. In Mammalian Protein Metabolism, pp. 21-132. Edited by H. M. Munro. New York: Academic Press.

Kimura, M. (1980). A simple method for estimating evolutionary rate of base substitutions through comparative studies of nucleotide sequences. J Mol Evol 16, 111-120.

Maddison, D. R. \& Maddison, W. P. (2000). MacClade: analysis of phylogeny and character evolution. Test version $4.0 \mathrm{~b} 12$. Sunderland, MA: Sinauer.

Montresor, M., Procaccini, G. \& Stoecker, D. K. (1999). Polarella glacialis, gen. nov. (Dinophyceae): Suessiaceae are still alive! J Phycol 35, 186-197.

Moreira, D., Le Guyader, H. \& Philippe, H. (2000). The origin of red algae and the evolution of chloroplasts. Nature 405, 69-72.

Posada, D. \& Crandall, K. A. (1998). Modeltest: testing the model of DNA substitution. Bioinformatics 14, 817-818.

Rodríguez, F., Oliver, J. F., Marín, A. \& Medina, J. R. (1990). The general stochastic model of nucleotide substitutions. $J$ Theor Biol 142, 485-501.

Silberman, J. D., Clark, C. G., Diamond, L. S. \& Sogin, M. L. (1999). Phylogeny of the genera Entamoeba and Endolimax as deduced from small subunit ribosomal RNA sequences. Mol Evol Biol 16, 1740-1751.

Stiller, J. W. \& Hall, B. D. (1999). Long-branch attraction and the rDNA model of early eukaryotic evolution. Mol Biol Evol 16, 1270-1279.

Swofford, D. L. (1998). PAUP* version 4.0b3. Sunderland, MA: Sinauer.

Tamura, K. \& Nei, M. (1993). Estimation of the number of nucleotide substitutions in the control region of mitochondrial DNA in humans and chimpanzees. Mol Biol Evol 10, 512-526.

Tippit, D. H. \& Pickett-Heaps, J. D. (1976). Apparent amitosis in the binucleate dinoflagellate Peridinium balticum. J Cell Sci $\mathbf{2 1}$, 273-289.

Tomas, R. N., Cox, E. R. \& Steidinger, K. A. (1973). Peridinium balticum (Levander) Lemmermann, an unusual dinoflagellate with a mesokaryotic and an eukaryotic nucleus. $J$ Phycol 9, 91-98.

Van de Peer, Y., Van der Auwera, G. \& De Wachter, R. (1996). The evolution of stramenopiles and alveolates as derived by "substitution rate calibration" of small ribosomal subunit RNA. $J$ Mol Evol 42, 201-210.

Van der Auwera, G., Hofmann, C. J., De Rijk, P. \& De Wachter, R. (1998). The origin of red algae and cryptomonad nucleomorphs: a comparative phylogeny based on small and large subunit rRNA sequences of Palmaria palmata, Gracilaria verrucosa, and the Guillardia theta nucleomorph. Mol Phylogenet Evol 10, 333-342.

Whitten, D. J. \& Hayhome, H. A. (1986). Comparative electrophoretic analysis of two binucleate dinoflagellates. J Phycol 22, 348-352. 